See discussions, stats, and author profiles for this publication at: https://www.researchgate.net/publication/260708059

\title{
Multigoal Heuristic Model Predictive Control Technique Applied to a Cascaded H-bridge StatCom
}

Article in IEEE Transactions on Power Electronics · March 2012

DOI: 10.1109/TPEL.2011.2165854

\section{CITATIONS}

84

3 authors:

Chris Townsend

University of Western Australia

56 PUBLICATIONS 559 CITATIONS

SEE PROFILE

R.E. Betz

University of Newcastle

156 PUBLICATIONS 2,426 CITATIONS

SEE PROFILE

Some of the authors of this publication are also working on these related projects:

Project Ironless Axial Flux Permanent Magnet Motor Drive View project

Energy Trading \& Electrical Stability in Decentralised Transactive Energy Markets View project
READS

49
T. J. Summers

University of Newcastle

83 PUBLICATIONS 832 CITATIONS

SEE PROFILE 


\title{
Multi-Goal Heuristic Model Predictive Control Technique applied to a Cascaded H-bridge StatCom
}

\author{
Mr Christopher D. Townsend ${ }^{1}$, Dr Terrence J. Summers ${ }^{2}$, \\ Dr Robert E. Betz ${ }^{3}$ \\ School of Electrical Engineering and Computer Science \\ University of Newcastle, Australia, 2308 \\ email: ${ }^{1}$ Christopher. Townsendenewcastle. edu. au \\ 2 Terry. Summers a newcastle.edu. au \\ ${ }^{3}$ Robert. Betz@newcastle. edu.au
}

\begin{abstract}
A multi-level H-bridge StatCom inherently contains redundancy in the available switching states. This paper develops a variation on the typical Model Predictive Control (MPC) scheme which is able to exploit this redundancy to simultaneously balance the H-bridge capacitor voltages, provide excellent current reference tracking and minimise converter switching losses. The scheme consists of a dead-beat current controller that has been integrated with heuristic models of the voltage balancing and switching loss characteristics. The integration of a Pulse Width Modulation (PWM) scheme is also described. Simulation and experimental results are presented that confirm the correct operation of the control and modulation strategies. Comparison with traditional control and modulation schemes is provided in terms of the key performance indicators associated with multi-level H-bridge StatComs.
\end{abstract}

Index Terms - Multi-level converters, STATCOM, voltage balancing, modulation strategy, control strategy.

\section{INTRODUCTION}

Multi-level Static Compensators (StatComs) are increasingly being implemented as cascaded H-bridge converters. The main reasons for the choice of this topology are: (i) the number of components used in the construction of each level scales linearly [1], [2] ; (ii) various control algorithms exist which control the balancing of capacitor voltages for high level numbers [3], [4]; (iii) the H-bridges form a modular section of each phase-leg which makes construction and maintenance easier; and (iv) the level number can be increased to allow direct connection of the converter to medium voltages which avoids the lossy and expensive connection transformer [5]. Throughout the remainder of this paper the acronym $\mathrm{H}$ StatCom will be used to refer to a multi-level StatCom implemented with cascaded H-bridges.

Traditional techniques for controlling the firing of the $\mathrm{H}-$ bridges in the individual phase-legs of a H-StatCom use either multiple triangular carrier waves for each of the bridges [6], [7] or precomputed firing angles for the bridges to implement Selective Harmonic Elimination (SHE) [8], [9]. The control and modulation techniques used in this paper are based on the instantaneous power concept [10], [11] coupled with deadbeat current control, heuristic models of voltage balancing and switching loss, and symmetrical PWM [12]. One advantage of this approach is that it operates in the stationary reference frame, and the control at any instant of time is dependent only on values measured or computed at that time. The deadbeat nature of the control loops means that their bandwidth is very high. In addition the calculations are numerically simple and ideal for digital implementation. Furthermore, it is relatively simple to incorporate a very high performance capacitor voltage balancing scheme into a MPC cost function. This scheme uses a sorting algorithm to evenly share the leg cluster voltage across the individual $\mathrm{H}$-bridge capacitors. The leg cluster voltage is defined as the sum of the capacitor voltages within a particular phase-leg. A separate control loop keeps the leg cluster voltages at a set-point value [13]. This technique avoids some of the control loop interaction problems present in other strategies [14].

In this paper the main indicators of performance for the control and modulation strategies will be the harmonic performance trade-off with the switching losses. A measure of performance for H-StatCom systems can be defined in various ways however many commercial $\mathrm{H}$-StatComs use the switching frequency per component relative to the harmonic performance as the basis for their design choice [15]. This is due to the fact that minimising the Total Harmonic Distortion (THD) and switching frequency results in a lower cost, smaller footprint design due to the reduced cooling requirements of the H-bridge modules.

This paper extends previous work by integrating a dead-beat current controller with heuristic models of the voltage balancing and switching loss characteristics of a $\mathrm{H}$-StatCom. The control approach is able to exploit the available redundancy in the switching states for a 9-bridge/leg H-StatCom to tradeoff between the capacitor voltage ripple and the amount of switching loss, while still achieving excellent reference current tracking performance. The simulation and experimental results presented demonstrate both the steady state and transient performance of the current tracking and voltage balancing characteristics. The performance of the MPC scheme in terms of reducing switching transitions is measured when the $\mathrm{H}-$ StatCom system is in a steady state operational condition.

Remark 1: The current trend in computing architectures is well suited to the implementation of MPC schemes. Modern 


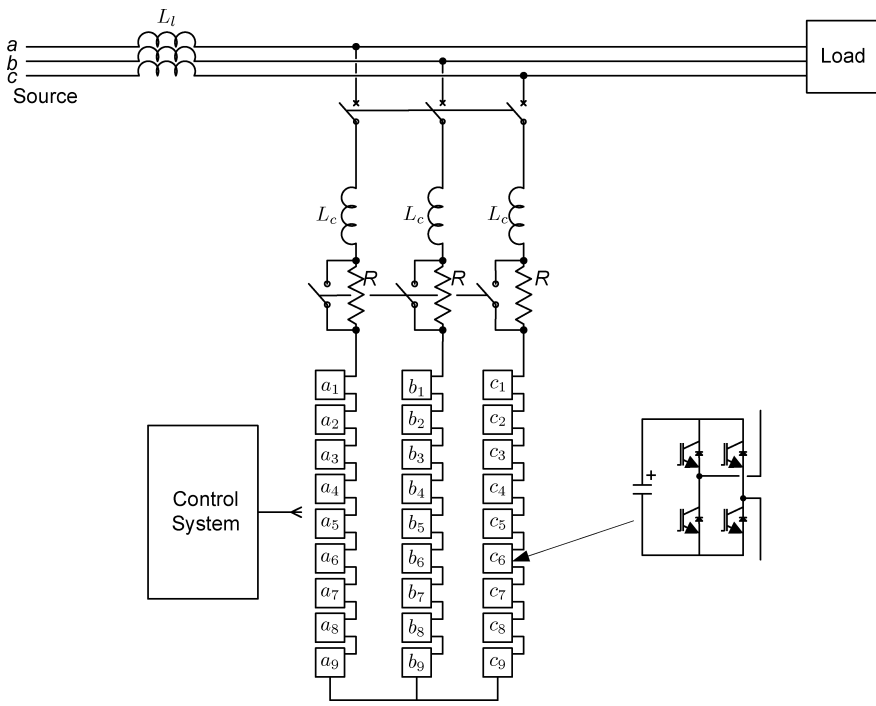

Figure 1. Circuit configuration of the star-connected 19-level H-bridge StatCom.

architectures are employing parallel computing to increase performance. This will be particularly advantageous in finite states MPC schemes where the increase in converter level number requires the evaluation of a large number of switching combinations that can be evaluated in parallel. As the level number increases, traditional modulation techniques such as Phase-Shifted Carrier (PSC) PWM will not scale as successfully due to their reliance on higher clock speeds.

\section{BACKGROUND}

Fig. 1 shows the circuit configuration for a 19-level (line to neutral) H-StatCom. Each stack of H-bridges essentially forms one phase of a three phase current controlled voltage source. The purpose of the H-StatCom is to modulate the voltage at the output of each stack so that the current through the inductors can be controlled to provide power factor correction, compensate for system harmonics, and alleviate other power quality problems.

Fig. 2 is a block diagram of the control scheme employed in this paper. Where $Q_{r}$ is the demanded reactive power, $P_{r}$ is the demanded real power, $\underline{i}_{r}^{Q}$ is the constituent reactive current component of the power, $\underline{i}_{r}^{P}$ is the constituent real current component of the power, $v_{r_{l e g}}$ is the target cluster voltage for each phase, $v_{l a}, v_{l b}$ and $v_{l c}$ are the measured cluster voltages for each phase, $\underline{i}_{r}$ is the reference current, $\underline{v}_{r_{l i m}}$ is the limited voltage generated by the dead-beat controller, $\underline{i}_{s c}$ is the measured H-StatCom current, $V_{d c_{1 . .27}}$ are the measured capacitor voltages, vector is the combination of capacitors that has been selected by the MPC scheme, $\underline{V}_{r e s}$ is the residual voltage difference between the dead-beat controller and the MPC controller and $\underline{v}_{g}$ is the measured supply voltage. The subscript _ is used to denote a vector quantity.

As can be seen from Fig. 2 the control is hierarchical, with the inner part of the structure executing the dead-beat current control and heuristic MPC scheme with the outer level re-

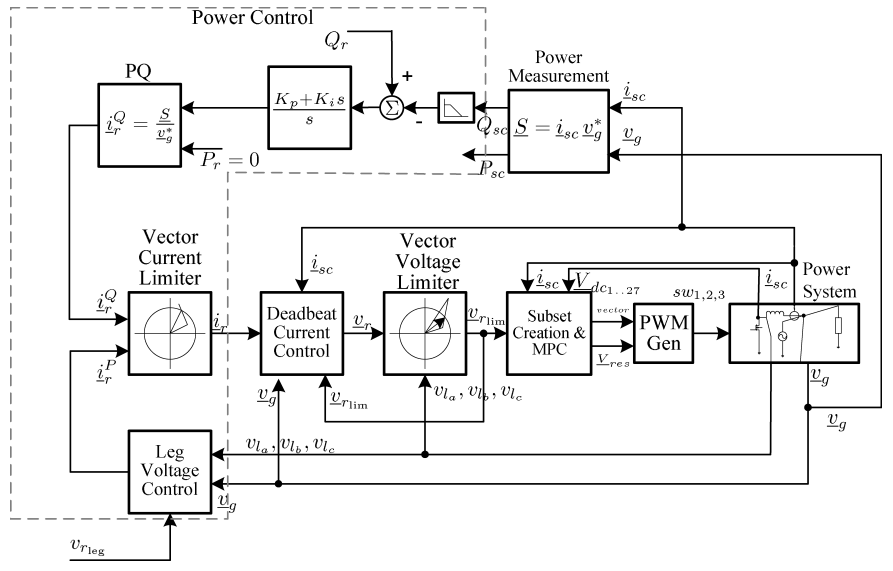

Figure 2. Block diagram of the H-StatCom control.

sponsible for the control of real and imaginary power. The PQ block uses instantaneous power theory [11] to decompose the reactive power into its constituent reactive current component. The reactive power is developed by a PI controller which acts on the difference between the demanded and measured reactive power. The presence of integral gain is needed to correct the steady state error in the reactive power. A particular source of steady state error is discussed in Section VIII.

The Leg Voltage Control block uses the component of the current associated with real power to balance the cluster voltages. This is achieved by normalising system voltage predictions (from a phase locked loop) and then scaling these values to form the desired component of the current associated with real power, the sign of the current being dependent on the error between the target and measured cluster voltages.

The current controller is based on a popular dead-beat current controller used in variable speed drive applications [12], [16]. This control strategy has a relatively high bandwidth compared to the PI current control technique used in other implementations of H-StatComs [14]. The dead-beat controller can track highly dynamic changes in the reference current within a few control cycles (depending on the magnitude of the DC bus voltages), and is relatively simple to implement. The basic equations used to calculate the required output $\mathrm{H}-$ StatCom voltage are shown in (1) and (2).

$$
\begin{gathered}
v_{k+1}^{r e f}=\frac{L}{T}\left(i_{k+1}^{r e f}-\hat{i}_{k}\right)+\hat{v}_{k+0.5}^{r e f} \\
\hat{i}_{k}=i_{k-1}+\frac{T}{L}\left(v_{k}-v_{k-0.5}^{s y s}\right)
\end{gathered}
$$

Where $i_{k-1}$ is the instantaneous sample of the current at time $t=(k-1) T$ and $i_{k+1}^{r e f}$ is the instantaneous value of the reference current at time $t=(k+1) T$. As for the voltage nomenclature, $v_{k}$ is the actual voltage applied during the interval from $(k-1) T \rightarrow k T, v_{k+1}^{r e f}$ is the desired (or reference) voltage that the controller applies from $k T \rightarrow(k+1) T$, $\hat{v}_{k+0.5}^{r e f}$ is the predicted instantaneous supply voltage at the midpoint of the control interval from $k T \rightarrow(k+1) T$ and $v_{k-0.5}^{s y s}$ is the measured instantaneous supply voltage at the 
midpoint of the control interval from $(k-1) T \rightarrow k T . T$ is the control period and $L$ is the connection inductance between the H-StatCom and the three-phase system. Note that (1) involves a projection of the reference current which is obtained using linear extrapolation. When the H-StatCom system is supplying unbalanced and/or harmonic loads the projection of the reference current can be improved using advanced phase locked loops [17].

The H-bridges chosen to make up the desired output voltage are crucial with respect to sharing the total cluster voltage evenly across the individual H-bridge capacitors. Before the heuristic MPC scheme was proposed, the balancing of the capacitors was achieved by carrying out an ordering of the capacitor voltages during the third quarter of each control period.

Remark 2: The sorting algorithm used in this paper creates an array of integers of size $N$ (where $N$ is the number of $\mathrm{H}-$ bridges in the phase-leg) to store the index of each capacitor voltage. The algorithm compares every two elements of the index array corresponding to the capacitor voltage that each index represents, and swaps the indexes if the first voltage is higher than the second. The first element of the resultant array of indexes will always point to the capacitor with the lowest voltage, with the final element pointing to the capacitor with the highest voltage. The execution time of this algorithm within the hardware system described in Section VI is less than 1 us when $N=9$.

The capacitor voltages, which are measured in the middle of a control period, are sorted in descending order of voltage if the instantaneous power flow is out of the phase-leg, and in ascending order if the power flow is into the phase-leg. Then, the number of capacitors required to create an output voltage less than or equal to the value of the desired output voltage is determined. This process starts at the top of the sorted queue of capacitor voltages. The residual voltage left over by this process will be of less magnitude than the next capacitor voltage. This residual voltage is then produced by using symmetrical PWM of the next H-bridge in the ordering. The other H-bridges selected in the process are switched so that their capacitor voltages are applied for the whole of the next control interval. Those that are not selected output the zero voltage. Using this algorithm the least charged capacitors are charged, and the most charged capacitors are discharged depending on the instantaneous power flow direction.

See [13] for a more detailed description of this control structure including the operation of the current and voltage limiters which prevent the StatCom from exceeding current limits and ensure that the $\mathrm{H}$-StatCom is capable of producing the desired output voltage.

Remark 3: Throughout the paper the term voltage vector is used to refer to the concept of each phase-leg producing a particular output voltage which forms one of the three directional components of a voltage space vector. In a H-StatCom system, there are a large number of switching combinations that create the same voltage vector. Within this paper the term switching combinations refers to the number of ways that the modulation scheme can create the voltage vectors by switching in or out various $\mathrm{H}$-bridges.

\section{The CONTROL AND MOdulation Structure}

The authors have included a new Subset Creation \& MPC block within the control structure shown in Fig.2. This block integrates the output of the dead-beat control equations with the previous capacitor voltage sorting algorithm which allows penalisation of the voltage balancing characteristics. A simple model of the switching losses is also included. The penalisation forms the basis of a cost function which selects the switching combination to be applied in the next control interval.

Information passed from the Subset Creation \& MPC block to the PWM Gen block includes the chosen switching combination and the residual voltage error between the voltage determined by the dead-beat controller and the voltage chosen (through selection of the switching states) by the MPC controller. The dead-beat control block has been kept within the control structure so that this residual voltage can be calculated. The PWM Gen block uses the residual error to calculate the required duty cycle to be applied to an appropriate bridge, using symmetrical PWM. The most appropriate bridge is determined by first sorting the capacitor voltages then selecting the $\mathrm{H}$ bridge with the lowest voltage (or highest voltage depending on the direction of instantaneous power flow) that has not already been switched in by the MPC scheme. Symmetrically pulse width modulating one of the $\mathrm{H}$-bridges within each phase-leg forms a hybrid scheme which has not been presented in previous applications of MPC [18], [19]. The hybrid scheme allows for the use of relatively low control frequencies, while still producing excellent current tracking performance due the modulation scheme's ability to apply a precise output voltage that subsequently controls the current. The use of lower control frequencies is important in a $\mathrm{H}$-StatCom of medium to high level number due to the significant computation time required to evaluate the MPC cost function.

Remark 4: It is unfeasible to utilise typical MPC schemes with readily available hardware in a medium to high level number H-StatCom. The relatively high control frequencies needed in these schemes to provide good current tracking performance coupled with the high number of required switching combinations to be evaluated (enforced by the need to balance the DC capacitor voltages) means the computational effort is often too large. This sub-optimal scheme using a conventional symmetrical PWM based current controller to generate the residual voltage is a reasonable approach to achieve good current tracking performance with a manageable computational burden.

Details of how the MPC scheme chooses which H-bridges to switch is presented in Section IV.

In typical finite state MPC applications there is one cost function which uses a system model to calculate an error for the particular switching combination being evaluated. For a 19-level H-StatCom there are more than one billion possible combinations that can be applied. Evaluating the error for 
such a large number of combinations requires significant computational effort which is unachievable within readily available hardware. The computational effort can be reduced by constraining the applied voltage vector to be within a subspace of the possible solution set [18], [20]. However even when the applied voltage vector is constrained as per [18], if capacitor voltage balancing is also a requirement then the number of combinations which need to be evaluated can exceed 15 million, depending on the magnitude of the voltage vector which needs to be applied and the number of capacitors needed to create this vector. In [18] the capacitor voltages are assumed to be maintained by separate DC supplies, therefore only the number of bridges that are switched in is of any relevance. When voltage balancing is included within the cost function, not only the number of bridges but also which bridges are switched becomes important. This means a greater number of combinations must be evaluated to find an acceptable solution. The authors have implemented three cost functions to reduce computation time. Each cost function evaluates the possible switching combinations for one phase-leg within the threephase system. The total number of switching combinations which can be applied in one phase-leg is $2^{N+1}-1$ [21], where $N$ is the number of H-bridges in the phase-leg. Therefore by evaluating the possible combinations on a per phase basis the total combinations reduce to $3\left(2^{N+1}-1\right)=3069$. It is feasible to evaluate this number of combinations within readily available hardware systems. Currently the authors have implemented the MPC scheme by utilising a powerful Pentium IV processor. However due to the simple nature of the heuristic models of the capacitor voltage balancing and switching losses it is feasible to evaluate this number of combinations in readily available dSPACE units and multi-core digital signal processors, particularly when a control frequency equal to or below $2.5 \mathrm{kHz}$ is utilised.

In Section $\mathrm{V}$ a comparison is provided in terms of the performance, between the MPC scheme developed in this paper and that developed by Cortes et al. in [18].

Utilising three independent cost functions may limit the performance of the MPC scheme as each cost function does not have the ability to consider the redundant voltage vectors across the three phases. This means the MPC scheme is unable to evaluate some redundant switching combinations which create the same voltage space vector and therefore control the three-phase currents in an identical fashion and possibly also provide a more optimal solution in terms of balancing the capacitor voltages and minimising the switching transitions. However it is shown in Sections VI and VII that the main limitation to further reducing switching transitions is the use of symmetrical PWM within the modulation scheme, not a lack of redundant switching combinations. Therefore it is unlikely that a cost function that considers the extra redundant switching combinations across the three phases will be able to reduce the switching transitions significantly more than the MPC scheme described in this paper.

\section{INTEGRATION OF DEAD-BEAT CURRENT CONTROL WITH THE HEURISTIC MPC SCHEME}

To control the H-StatCom current the scheme described in this paper integrates the output of a dead-beat current controller with a heuristic MPC scheme. To describe this implementation firstly the relationship between the switching combinations and the corresponding voltage vectors is formalised:

$$
v_{k+1}^{a p p}=V_{d c} \bullet S_{i}
$$

where $v_{k+1}^{a p p}$ is the applied voltage vector generated by the switching states $S_{i}$, for the interval from $k T \rightarrow(k+1) T$.

Each H-bridge in the phase-leg is capable of producing three output voltages, $+V_{d c},-V_{d c}$ and $0 V$. Therefore when evaluating (3) there are a total of $2^{N+1}-1$ voltage vectors which can be applied at the output of each phase. For each $\mathrm{H}$-bridge, there is only one switching combination to create each of the $+V_{d c}$ and $-V_{d c}$ voltages and two possible combinations to create the $0 \mathrm{~V}$ output voltage. The deadbeat current controller used in this paper does not consider the redundancy in creating the zero voltage output vector. The redundancy is however considered within the modulation scheme which modifies which switching combination is used to create the zero voltage output depending on whether the output voltage is in the positive or negative half cycle. Utilising this technique during steady state operation of the H-StatCom means that both legs of a $\mathrm{H}$-bridge module only undergo switching transitions during zero volt crossings. This reduces the total number of required switching transitions within each H-Bridge module.

The Subset Creation \& MPC block shown in Fig. 2 firstly evaluates the corresponding voltage vector for each switching combination using (3). By comparing to the output of the deadbeat current controller, combinations that produce a residual voltage greater than the lowest capacitor voltage are disregarded, hence creating a subset of the available combinations. This evaluation is performed by disregarding any combinations that do not satisfy the condition in (4).

$$
\left|v_{k+1}^{r e f}-v_{k+1}^{a p p}\right|<V_{d c, \text { min }}
$$

where $V_{d c, \text { min }}$ is the minimum capacitor voltage within the phase-leg and $v_{k+1}^{r e f}$ is the reference voltage (calculated within the dead-beat controller) that needs to be applied from $k T \rightarrow$ $(k+1) T$.

Remark 5: This means the implementation of the current tracking model in this paper differs from typical MPC applications, with this implementation resembling more of a search for combinations that provide satisfactory current tracking performance.

An MPC cost function is created to include heuristic models of the voltage balancing and switching loss characteristics. The form of the cost function is:

$$
\text { error }=\alpha_{1}\left(V_{\text {cap,error }}\right)+\alpha_{2}\left(S W_{\text {transitions }}\right)
$$


where $S W_{\text {transitions }}$ is the number of transitions from the currently applied switching combination to the evaluated combination and $V_{\text {cap,error }}$ is a measure of how far the evaluated combination will drive the capacitor voltages either towards or further from their target value.

To evaluate (5) the Subset Creation \& MPC block shown in Fig. 2 executes the following steps for each possible switching combination that has been deemed satisfactory through the evaluation of (4):

1) Apply the voltage balancing penalisation by executing the following steps:

a) Sort the capacitor voltages from highest to lowest;

b) If power is flowing into the phase-leg then reverse the order of the capacitor voltages;

c) Create an array of $N$ integers, where $N$ is the number of H-bridges in each phase-leg. The first element in the array contains an $N$ bit number corresponding to the first capacitor which needs to be switched in. E.g. if power is flowing into the stack of a nine-bridge phase-leg, and capacitor number three has the lowest voltage then the first element of the array will be 000000100 . In this example if capacitor number eight has the highest voltage then the ninth element of the array will be 010000000;

d) Create an $N$ bit number for each possible switching combination. Continuing the example, if capacitors one and four are switched in for a particular combination, while the others are switched out then the corresponding number will be 000001001 ;

e) Upon the evaluation of each switching combination, logically AND the $N$ bit number created in Step (d) with each element of the array created in Step (c). If the logical operation results in a non-zero number then accumulate an error value which is modulated based on the need for the evaluated capacitor to be switched in. Continuing the example, if the evaluated switching combination is 000001001 and capacitors one and four have the lowest voltages, and power is flowing into the stack then the resultant error will be zero (as this is the ideal solution). However if power was flowing out of the stack the error would be $9+8=17$. The higher the error the less likely that switching combination will be applied in the next control interval.

2) Compare the switching combination under investigation to the combination that is currently being applied. An error value that penalises switching transitions is obtained by counting the number of non-zero elements in the $S W_{\text {transitions }}$ array after the element-wise subtraction performed in (6).

$$
S W_{\text {transitions }}=s w_{k}-s w_{k+1}
$$

where,

$$
s w=\left[\text { bridge }_{1}, \text { bridge }_{2} \ldots \text { bridge } e_{N}\right]
$$

bridge $_{i}$ can take the values $+1,-1$ or 0 representing the possible output voltages from the $i^{\text {th }} \mathrm{H}$-bridge module during either the interval from $(k-1) T \rightarrow k T$ or $k T \rightarrow(k+1) T$ (indicated by the $k$ or $k+1$ subscript respectively);

3) Multiply the two error components by different scaling factors before they are summed in the cost function to give a total error.

The above process is carried out for all the switching combinations and the combination with the lowest error is applied in the next control interval.

The scaling factors used in the scheme presented in this paper are chosen based on the following rationale: the model of the current tracking does not affect the average volt seconds applied within a control interval, provided that the residual voltage error is less than the lowest capacitor voltage. This condition is enforced within the scheme by specifically excluding switching combinations for which this condition is not true. The total applied volt seconds is always precise due to the residual voltage being applied via the symmetrical PWM.

When the performance of the current tracking is guaranteed, the scaling factors can be obtained empirically. When the scaling factor for the voltage balancing model is non-zero and the switching loss scaling factor is set to zero, the voltage balancing will dominate the cost function and give the tightest control over capacitor voltage ripple. A non-zero setting for the switching loss scaling factor reduces device switching. As this value increases at some point the voltage ripple on the capacitors will become unacceptable. The maximum capacitor voltage ripple will be dependent on the chosen capacitors and the effect of voltage ripple on their lifetime. Further manipulation of the scaling factors is discussed in Section VII.

There are various empirical methods to calculate the optimum scaling factors [22]. One method that could be adapted to the MPC scheme described in this paper is an off-line optimisation technique which is based on the running of large numbers of simulations. The simulation results are used to measure the performance of each set of scaling factors in terms of a defined set of merits [23]. Using the intersection of regions of space that quantify the merits it is possible to select scaling factors that provide close to optimal performance for each of the models within the cost function.

The prediction horizon for the MPC scheme used in this paper is one. This means that the switching combination chosen for each control interval is based only on events which occur in the models within one control cycle. There are advantages in increasing the prediction horizon, namely that with longer predictions of the H-StatCom current it is possible to switch in capacitors that are less likely to exceed their voltage bounds in the near future. This will allow each capacitor to be switched in for longer periods and therefore further reduce switching losses. However, there is an exponential relationship between the prediction horizon and the number of 
switching combinations that need to be evaluated. This means that it is often difficult to increase prediction horizons for relatively high level number $\mathrm{H}$-StatComs, where the number of combinations are already substantial.

Due to computation delay it is of course non-causal to evaluate the control algorithm in the same interval in which the control action is to be applied. Therefore when the control algorithm is being executed in the control interval from $(k-1) T \rightarrow k T$ prediction of the H-StatCom current is performed to calculate the value of the current at $t=k T$. This prediction is performed by evaluating (2). The heuristic MPC scheme used in this paper does not predict the value of the capacitor voltages at $t=k T$. These voltages are sampled at the midpoint of each control interval therefore the expected error between the sampled value and actual value at $t=k T$ is relatively small. To improve the scheme it is possible to calculate the change in capacitor voltages over the half control cycle with knowledge of the current magnitude and direction and H-bridge capacitance value.

The switching model has full knowledge of the switching states at $t=k T$ due to the selection of these states in the previous interval. The state of the H-bridge that is pulse width modulated is also known due the scheme utilising symmetrical PWM. Therefore computation delay does not affect the model of the switching losses within the cost function.

\section{Simulation Results}

A simulation of the scheme has been implemented in Saber ${ }^{\circledR}$ and is very accurate and comprehensive. It completely simulates the H-bridge phase-legs, the start-up sequencing and all control loops. It is a multi-mode simulation, with the control implemented digitally with the timing as per the experimental system. Each part of the control scheme is implemented as ' $\mathrm{C}$ ' DLLs, and this code is also used in the actual experimental system.

Fig. 3 shows waveforms for a transient condition within the H-StatCom system. From $0.5 \mathrm{~s}$ to $0.55 \mathrm{~s}$ the H-StatCom is absorbing inductive VARs. At this point a transient from 4KVARs inductive to 4KVARs capacitive occurs. Fig. 3 shows the 'a' phase H-StatCom current (bottom plot), supply voltage (top plot), stack voltage (top plot) and the nine capacitor voltages for phase 'a' (middle plot). The expanded window within Fig. 3 shows a close up of the capacitor voltages.

The system has a high bandwidth, with the change of state occurring in one control interval $(400 \mu s)$. This achieves the same performance as the dead-beat control previously implemented on this system [13]. Performing an FFT on the current when the H-StatCom is absorbing 4KVARs capacitive gives a THD of $1.7 \%$.

The capacitor voltages all have the normal $100 \mathrm{~Hz}$ ripple component present in $\mathrm{H}$-StatCom systems, however the deviation from the mean DC value is increased. The heuristic MPC models allow increased voltage excursions away from the mean DC value by penalising the switching transitions in the cost function. A detailed discussion regarding the trade-offs

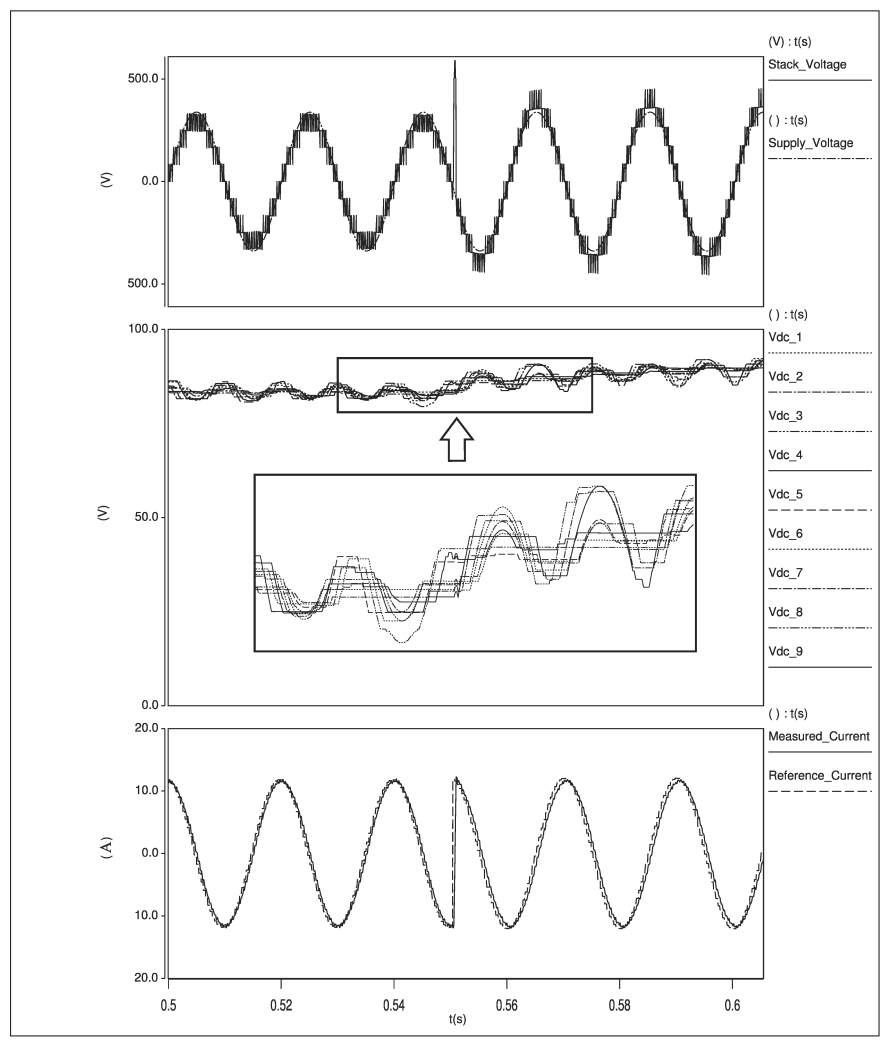

Figure 3. Simulation waveforms for scaling factors $\alpha_{1}=0.05$ and $\alpha_{2}=0.2$ - Top plot: stack and supply voltages, Middle plot: nine H-bridge capacitor voltages, Bottom plot: H-StatCom reference and measured current.

between the number of switching transitions and the increased ripple in the capacitor voltages is provided in Section VII.

Fig. 4 demonstrates the current tracking performance of an MPC scheme similar to that developed by Cortes et al. in [18]. The scheme limits the subspace of switching combinations to only allow progression of the voltage, in the next control interval, to an adjacent vector. The waveforms show that the current tracking is good when the system is in steady state. However under transient conditions it is possible that the current experiences an overshoot as seen in Fig. 4. The overshoot occurs as a result of the voltage increasing positively to drive the current to its new reference value, however once the current reaches the reference, the voltage vector ideally needs to have a negative polarity. Due to the limited subspace evaluation, the voltage takes a finite period to reach the ideal vector that the dead-beat controller has calculated and therefore current overshoot occurs. This differs from the results in Fig. 3 which show that by evaluating the entire set of switching combinations the dynamic performance is only limited by the control frequency and the chosen DC capacitor voltage.

\section{EXPERIMENTAL RESULTS}

In order to validate the simulation studies, the ' $C$ ' code dll used in the Saber simulation was slightly modified for operation in the real-time control environment of a low volt- 


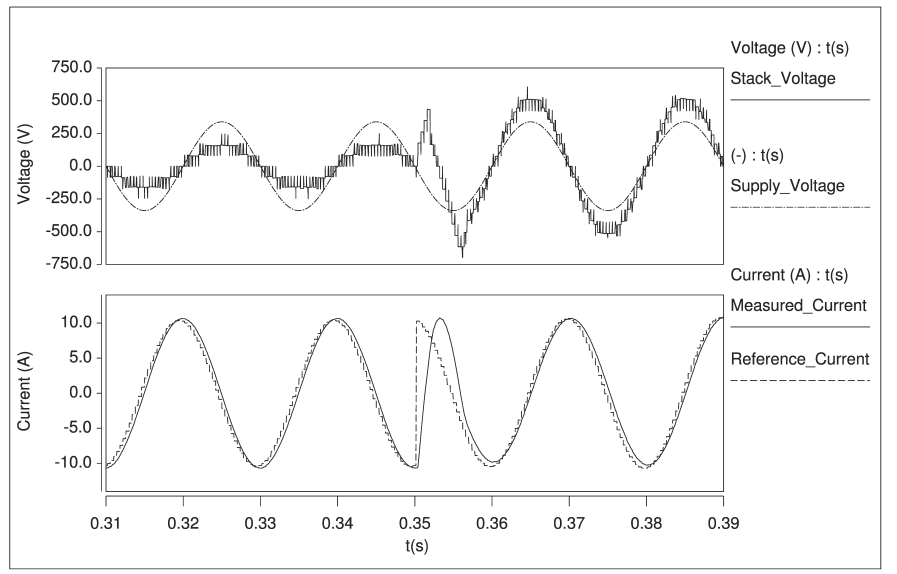

Figure 4. Simulation waveforms when evaluating a limited subspace of voltage vectors - Top plot: stack and supply voltages, Bottom plot: H-StatCom reference and measured current.

age (415VAC) 19-level H-StatCom. The H-StatCom used to produce the experimental results is a scaled model of an $11 \mathrm{kV}$ $\mathrm{H}$-StatCom. It has $9 \mathrm{H}$-bridges per phase, with each $\mathrm{H}$-bridge designed with MOSFET power devices. The phase-legs are Wye connected. A block diagram of the experimental system appears in Fig. 5. One can see that it is implemented as a multiprocessor system, with individual processors implementing the control for each of the phase-legs. These phase-leg processors are responsible for switching in the desired capacitors and applying the PWM. The desired switching combinations which are passed to the phase-leg controllers are developed in the MPC scheme, which is implemented in a central Pentium IV PC. The experimental system uses a control frequency of $2.5 \mathrm{kHz}$. The corresponding $400 \mu \mathrm{s}$ control interval is divided into four equal sections. The Pentium IV processor executes the control algorithm within the third quarter of the control interval. This is necessary due to the supply voltage being sampled at the midpoint of the control interval. To increase the available execution time the voltage can be sampled at the beginning of the control interval with appropriate modification of (2). To execute the control scheme described in this paper the Pentium IV processor requires approximately $90 \mu \mathrm{s}$.

Fig. 6 shows the actual 'a' phase H-StatCom current and the reference current on the top plot, and the nine capacitor voltages for phase 'a' on the bottom plot, for the start-up condition within the $\mathrm{H}$-StatCom. At $0.04 \mathrm{~s}$ the $\mathrm{H}$-StatCom capacitors have been charged sufficiently and the control scheme begins producing currents to absorb 1.3KVARs inductive. It can be seen in Fig. 6 that the system achieves a good current tracking performance with tight control over the capacitor voltages. The capacitor voltage ripple is similar to that achieved by a previous system described in [13]. This is due to the fact that the switching loss penalty was set to zero to obtain these results, and therefore the heuristic MPC models are selecting the same switching combinations as in [13]. The previous scheme used only dead-beat current control coupled with the capacitor balancing scheme described in Section II and there-

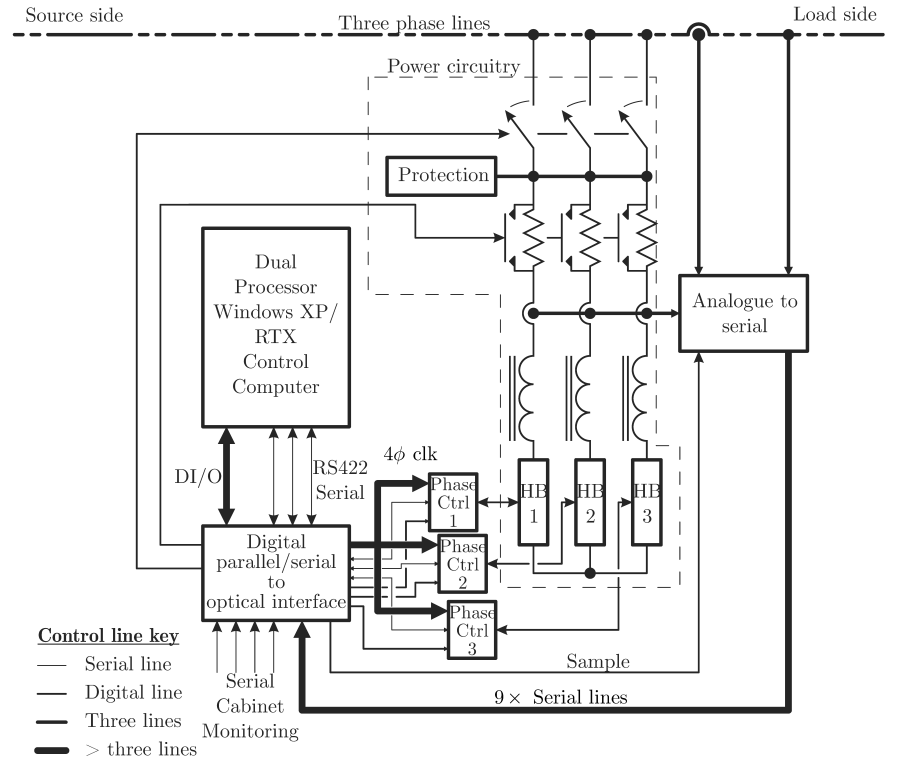

Figure 5. Block diagram of the 415-V 10-kVA experimental system.
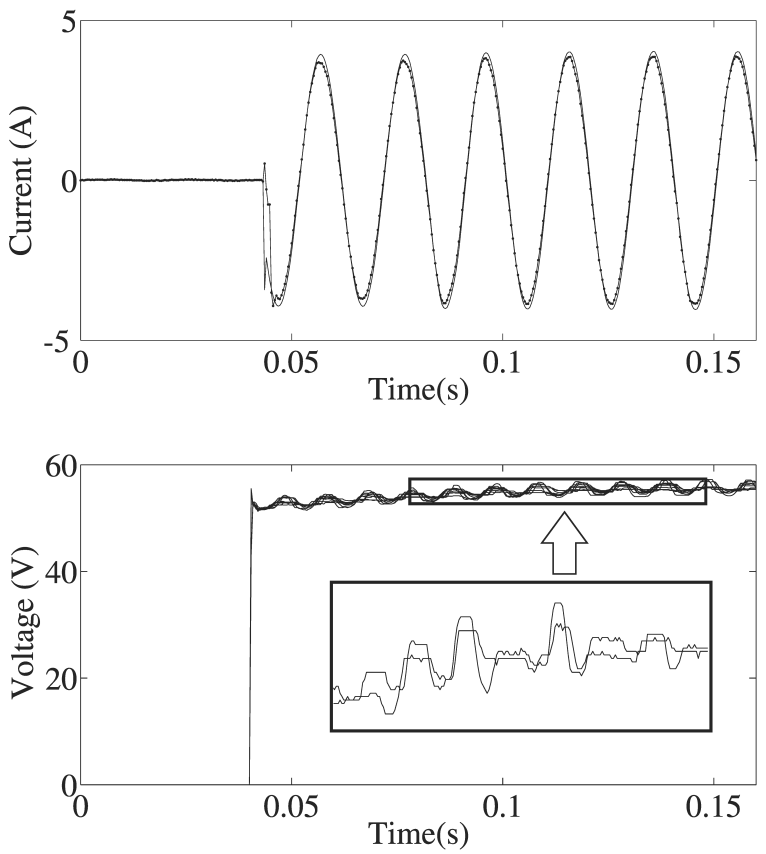

Figure 6. Experimental waveforms for scaling factors $\alpha_{1}=0.05$ and $\alpha_{2}=$ 0.0 - Top plot: H-StatCom reference and measured current, Bottom plot: nine $\mathrm{H}$-bridge capacitor voltages.

fore could not trade-off the voltage balancing characteristics with the switching losses. The expanded window within Fig. 6 shows a close up of two of the capacitor voltages. It can be seen that each capacitor experiences a similar voltage ripple.

Fig. 7 shows waveforms for a transient condition within the $\mathrm{H}$-StatCom system similar to Fig. 3. Initially the H-StatCom is absorbing inductive VARS. At approximately $0.02 \mathrm{~s}$ a transient 

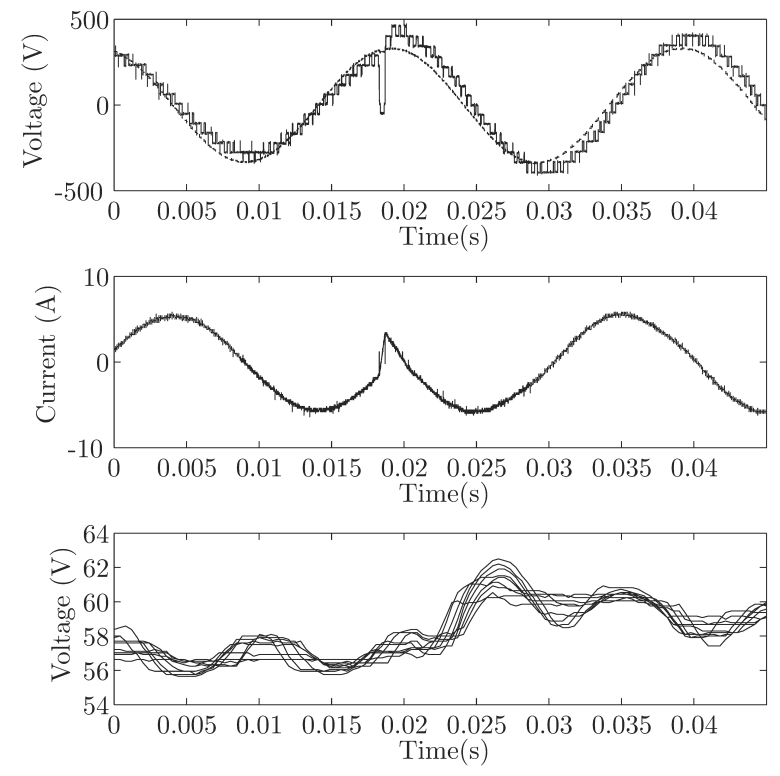

Figure 7. Experimental waveforms for scaling factors $\alpha_{1}=0.05$ and $\alpha_{2}=$ 0.1 - Top plot: stack and supply voltages, Middle plot: H-StatCom measured current, Bottom plot: nine H-bridge capacitor voltages.

from 1.3KVARs inductive to 1.3KVARs capacitive occurs. Fig. 7 shows the 'a' phase H-StatCom current (middle plot), line voltage (top plot), stack voltage (top plot) and the nine capacitor voltages for phase 'a' (bottom plot).

Fig. 7 confirms the high bandwidth of the system with the change in state occurring in one control interval $(400 \mu s)$. There is excellent correspondence between this experiment and the simulated output shown in Fig. 3.

Fig. 8 shows the re-convergence of the capacitor voltages after the capacitors have initially undergone unequal charging periods. The control scheme begins operating at approximately $0.04 \mathrm{~s}$ and within two cycles the voltages converge to the DC mean value. The scheme described in [13] is able to force convergence of the capacitor voltages in approximately $75 \%$ of the time it takes the heuristic MPC model of the voltage balancing described in this paper. The use of the heuristic MPC model requires longer for re-convergence due to the penalisation of the switching transitions within the cost function which acts to maintain the switching state of each $\mathrm{H}$-bridge module, even in the presence of capacitor voltage unbalance.

Fig. 9 shows the 'a' phase H-StatCom current, reference current (top plot) and the nine capacitor voltages for phase 'a' (bottom plot) when the H-StatCom is absorbing 1KVAR inductive. The switching loss penalty within the heuristic MPC scheme has been set at 0.5 to obtain these results. In Fig. 9 it can be seen that the system still achieves good current reference tracking performance with an increased capacitor voltage ripple compared to Fig.s 6 and 7. While the system is still achieving capacitor voltage balancing, by penalising the

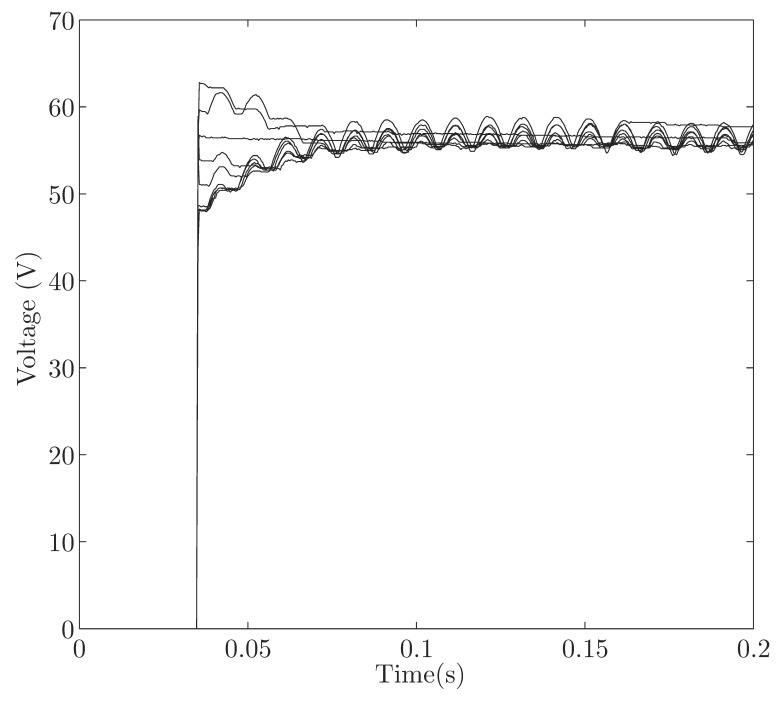

Figure 8. Experimental waveforms for scaling factors $\alpha_{1}=0.05$ and $\alpha_{2}=$ 0.4 depicting the convergence of the nine $\mathrm{H}$-StatCom capacitor voltages.

\begin{tabular}{|c|c|c|c|}
\hline $\begin{array}{c}\text { Voltage } \\
\text { Balancing } \\
\text { Scaling Factor }\end{array}$ & $\begin{array}{c}\text { Switching loss } \\
\text { Scaling Factor }\end{array}$ & $\begin{array}{c}\text { Maximum } \\
\text { Capacitor } \\
\text { Ripple(V) }\end{array}$ & $\begin{array}{c}\text { Switch } \\
\text { Transitions / } \\
\text { Component }\end{array}$ \\
\hline 0.05 & 0 & 3.2 & 3075 \\
\hline 0.05 & 0.1 & 3.2 & 2827 \\
\hline 0.05 & 0.2 & 3.9 & 2597 \\
\hline 0.05 & 0.3 & 4.4 & 2420 \\
\hline 0.05 & 0.4 & 5 & 2340 \\
\hline 0.05 & 0.5 & 5.6 & 2260 \\
\hline 0.05 & 0.8 & 6.5 & 2195 \\
\hline 0.05 & 1.1 & 9.1 & 2172 \\
\hline
\end{tabular}

VOLTAGE RIPPLE AND SWITCHING LOSS TRADE-OFF.

number of switching transitions the control scheme is able to reduce the switching losses at the expense of capacitor voltage ripple. The expanded window within Fig. 9 shows a close up of two of the capacitor voltages. It can be seen that one of the capacitors experiences a larger voltage ripple than the other. This is a direct consequence of the heuristic MPC scheme keeping this H-bridge switched in for an extended period so as to reduce switching losses.

To quantify the amount of reduction which can be achieved by employing this control structure, varying penalties were applied to the switching component of the MPC cost function over a $3.2 \mathrm{~s}$ period. The results of this experimentation are shown in Table I. These results are compared to a traditional modulation scheme for a H-StatCom in Section VII.

\section{DISCUSSION}

It is possible to investigate the trade-off between harmonic performance and switching losses for the control scheme developed in this paper. This can be done in various ways however many commercial H-StatComs use the switching frequency per component as the basis for their design choice [15], [24]. Traditionally the best trade-off between harmonic 

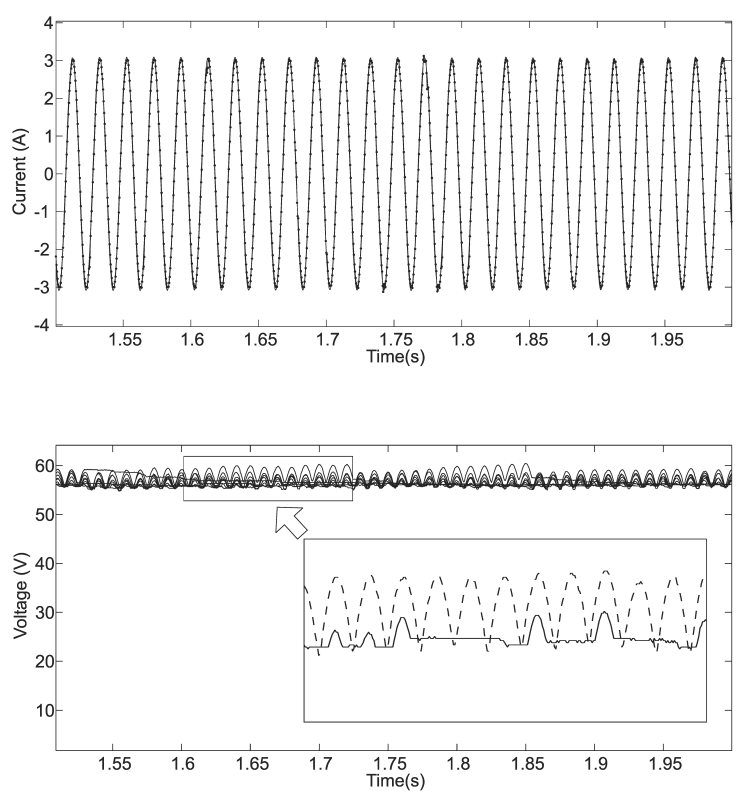

Figure 9. Experimental waveforms for scaling factors $\alpha_{1}=0.05$ and $\alpha_{2}=0.5$ with increased penalisation of switching losses - Top plot: $\mathrm{H}-$ StatCom reference and measured current, Bottom plot: nine H-bridge capacitor voltages.

performance and switching loss has been achieved using PSCPWM. By developing a H-StatCom simulation that implements the PSC-PWM scheme described in [14], it is possible to calculate the theoretical values of total harmonic distortion which can be achieved using this modulation strategy.

It was noted in Section V that the THD of the H-StatCom current, when utilising the scheme described in this paper, was measured at $1.7 \%$. To equal this harmonic performance for the same operational condition (absorbing 4KVARs capacitive), the PSC-PWM scheme requires a carrier frequency of $250 \mathrm{~Hz}$. For PSC-PWM each switching device will undergo two switching transitions per period of the carrier waveform, this is due to the fact that PSC-PWM switches in each $\mathrm{H}$ bridge during every period of the carrier waveform. This means the total switching transitions per component for a $3.2 \mathrm{~s}$ period of time will be 1600 .

The number of transitions as a function of MPC cost function switching penalty is shown graphically in Fig. 10 . The number of transitions for a PSC-PWM scheme is shown to provide a comparison.

Remark 6: To provide a comparison between the author's scheme and PSC-PWM the voltage ripple, and it's effect on capacitor lifetime, must also be considered by the system designer. With identical H-StatCom currents, the implementation of PSC-PWM will result in a different capacitor voltage ripple to that of the scheme described in this paper. This is due to the differing pulse widths which result when using PSCPWM. When creating a large stack voltage i.e. capacitive

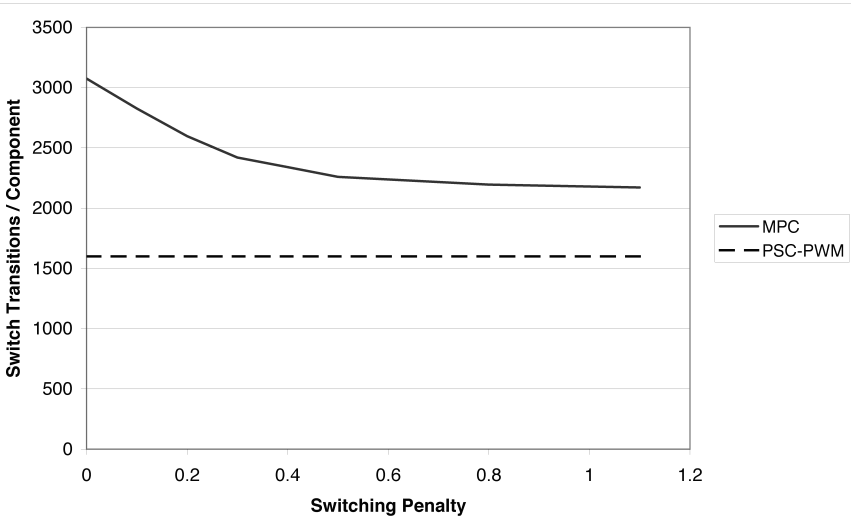

Figure 10. Switching transitions as a function of switching loss scaling factor.

currents, the pulse widths for all bridges become larger and therefore the ripple will increase. For small stack voltages i.e. inductive currents, the pulse widths for all bridges become smaller and therefore the ripple will decrease. For comparison purposes, the ripple produced in the PSC-PWM scheme can be considered to be similar to that of the case of zero switching loss penalty. This is the case in which the only component of the ripple is the normal $100 \mathrm{~Hz}$ component traditionally seen in $\mathrm{H}$-StatCom systems.

The experimental results show that the heuristic MPC scheme can significantly reduce the switching transitions up to a certain point. The shape of the plot shown in Fig. 10 indicates that as the switching penalty increases beyond 0.5 the rate at which the transitions reduce starts to decrease. The system is reaching a limit imposed by the fact that a minimum number of transitions need to occur to allow creation of the basic shape of the voltage waveform. The heuristic MPC scheme must switch in enough capacitors so that the PWM can create the extra volt seconds to accurately track the reference current. As only one bridge is pulse width modulated a minimum number of capacitors must be switched in to create the majority of the output stack voltage, as the sinusoidal shape of the waveform progresses.

\section{CONTRIBUTIONS \& CONCLUSIONS}

This paper presents a variation on the typical MPC control scheme for a H-StatCom which provides excellent current tracking performance while simultaneously trading off the voltage balancing characteristics with the switching losses. This scheme is integrated with a novel hybrid of deadbeat current control, heuristic MPC and a PWM scheme. Comprehensive simulation and experimental results showing the performance of the schemes have been presented. The results have important implications for the trade-offs between schemes based on symmetrical PWM and phase-shifted carrier PWM. The switching frequency per component of the MPC scheme was not shown to be less than that of PSC-PWM. However, when considering the harmonic degradation of the practical implementation of PSC-PWM schemes [25], the use 
of heuristic MPC will allow the performance of symmetrical PWM schemes to exceed that of PSC-PWM, in some $\mathrm{H}$ StatCom applications. Therefore, it is proposed that with the ease of practical implementation of symmetrical PWM this technique will begin to gain wider acceptance for use in $\mathrm{H}$ StatComs above a certain level number.

\section{A. Future Work}

The application of dead-beat current control coupled with the capacitor voltage balancing techniques described in this paper have been seen to be very stable over a wide range of operating conditions. However future work will focus on developing a formal stability analysis of the presented heuristic MPC scheme. The scheme has been designed to ensure that the current tracking performance is identical to that of the well known dead-beat current controllers described in [12], [26], [27]. The stability of dead-beat current control has been confirmed in [28] where the assumption is made that there always exists a DC bus voltage within the inverter that is capable of producing the appropriate output voltage. Therefore to confirm the stability of the heuristic MPC scheme all that remains to be shown is that the capacitor voltages remain balanced and bounded within a certain range so that they are capable of producing the output voltage. In a further paper the authors will use a sampled-data model to develop a formal proof of the voltage balancing.

It was mentioned in Section II that a PI loop acting on the reactive power is needed to correct for any open loop error in the reactive power tracking performance. One significant source of error arises due to the presence of a large number of series connected switches and/or diodes in the H-StatCom topology which means that there is a significant cumulative voltage drop across the devices. For example, typical voltage drops across a switched on IGBT of $1.7 \mathrm{kV}$ rating is of the order of 2 Volts or more. Since there is the possibility of two switches being in the conduction path this means that there could be a voltage drop of up to 18 volts for a 9 bridge phase-leg. Even if the H-bridges are commanded to output zero voltages the actual voltage at the terminals of each H-bridge will be the voltage drop of one switch and one diode. The cumulative voltage drops cause errors in the current tracking performance that are exacerbated in any H-StatCom system which uses a predictive current controller, such as that employed in this paper. A compensation algorithm has been developed in [29] which uses a feed-forward control structure to modify the applied phase-leg voltage and eliminate the error in the reactive power tracking. With some modification the scheme described in [29] can be integrated into the MPC algorithm by modifying the ideal voltage vector used to evaluate each switching combination. This will be the subject of future work.

It has been shown that MPC allows a trade-off between capacitor voltage ripple and the number of switching transitions. The MPC scheme can be modified to improve this tradeoff and reduce the voltage excursions without significantly increasing the switching transitions. This involves modifying the model of the voltage balancing used within the cost function. Instead of only sorting the capacitor voltages, the relative voltage difference between each capacitor and the mean DC value can also be penalised. This should decrease the number of voltage excursions outside the normal $100 \mathrm{~Hz}$ ripple. To further improve the trade-off, the scaling factors within the cost function can be changed dynamically depending on where in the voltage and current waveform the next control interval resides. Future work will aim to implement these techniques which will further increase the performance of symmetrical PWM relative to the traditional phase-shifted carrier modulation schemes.

\section{REFERENCES}

[1] J. Rodriguez, J. S. Lai, and F. Z. Peng, "Multilevel inverters: A survey of topologies, controls, and applications," IEEE Transactions on Industrial Electronics, vol. 49, pp. 724-738, August 2002.

[2] H. Iman-Eini, J.-L. Schanen, S. Farhangi, and J. Roudet, "A modular strategy for control and voltage balancing of cascaded h-bridge rectifiers," Power Electronics, IEEE Transactions on, vol. 23, no. 5, pp. 2428 $-2442,2008$

[3] T. Yoshii, S. Inoue, and H. Akagi, "Control and performance of a medium-voltage transformerless cascade pwm statcom with starconfiguration," in Industry Applications Conference, 2006. 41st IAS Annual Meeting. Conference Record of the 2006 IEEE, vol. 4, pp. 1716 $-1723,2006$

[4] D. Gerry, P. Wheeler, and J. Clare, "A voltage-balance strategy for multilevel, multi-cellular converters," in CDROM Proceedings of the 10th EPE, September 2003. ISBN 90-75815-07-7.

[5] Q. Song and W. Liu, "Control of a cascade statcom with star configuration under unbalanced conditions," Power Electronics, IEEE Transactions on, vol. 24, no. 1, pp. $45-58,2009$.

[6] J. Ning and Y. He, "Phase-shifted suboptimal pulse-width modulation strategy for multilevel inverter," in Industrial Electronics and Applications, 2006 1ST IEEE Conference on, (Singapore), pp. 1-5, May 2006.

[7] R. Naderi and A. Rahmati, "Phase-shifted carrier pwm technique for general cascaded inverters," Power Electronics, IEEE Transactions on vol. 23, pp. $1257-1269$, may. 2008.

[8] Q. Song, W. Liu, and Z. Yuan, "Multilevel optimal modulation and dynamic control strategies for statcoms using cascaded multilevel inverters," Power Delivery, IEEE Transactions on, vol. 22, pp. $1937-1946$, jul. 2007.

[9] A. Cetin and M. Ermis, "Vsc-based d-statcom with selective harmonic elimination," Industry Applications, IEEE Transactions on, vol. 45, pp. $1000-1015$, may. 2009.

[10] H. Akagi, Y. Kanazawa, and A. Nabae, "Instantaneous reactive power compensators comprising switching devices without energy storage components," IEEE Transaction on Industry Applications, vol. 20, pp. 625 630, May/June 1984

[11] H. Akagi, E. H. Watanabe, and M. Aredes, Instantaneous Power Theory and Application to Power Conditioning. John Wiley and Sons, 2007.

[12] R. Betz, B. Cook, and S. Henriksen, "A digital current controller for three phase voltage source inverters," Conference Record of the IEEEIAS Annual Meeting, pp. 722-729, New Orleans, Oct. 1997.

[13] R. E. Betz, T. Summers, and B. Cook., "Outline of the control design for a cascaded h-bridge statcom," in Proceedings of the IEEE-IAS Annual Meeting, Edmonton Canada, Oct 2008. ISBN 978-1-4244-2279-1.

[14] H. Akagi, S. Inoue, and T. Yoshii, "Control and performance of a transformerless cascade pwm statcom with star configuration," Industry Applications, IEEE Transactions on, vol. 43, pp. 1041 -1049, jul. 2007.

[15] C. Davidson and G. de PrÈville, "The future of high power electronics in transmission and distribution power systems," in Power Electronics and Applications, 2009. EPE '09. 13th European Conference on, pp. 1-14, Sept. 2009.

[16] D. G. Holmes and D. A. Martin, "Implementation of a direct digital predictive current controller for single and three phase voltage source inverters," in Industry Applications Conference, 1996. Thirty-First IAS Annual Meeting, IAS '96., Conference Record of the 1996 IEEE, vol. 2, (San Diego, CA), pp. 906-913, Oct. 1996. 
[17] R. Betz, C. Townsend, T. Summers, and G. Mirzaeva, "Simultaneous estimation of sequence and harmonic components for statcom applications," in Power Electronics and Motion Control Conference (EPE/PEMC), 2010 14th International, pp. T7-47 -T7-54, 2010.

[18] P. Cortes and A. Wilson and S. Kouro and J. Rodriguez and H. Abu-Rub, "Model predictive control of multilevel cascaded h-bridge inverters," Industrial Electronics, IEEE Transactions on, vol. 57, pp. 2691 -2699, aug. 2010.

[19] P. Lezana, R. Aguilera, and D. Quevedo, "Model predictive control of an asymmetric flying capacitor converter," Industrial Electronics, IEEE Transactions on, vol. 56, pp. 1839 -1846, june 2009.

[20] M. Perez, P. Cortes, and J. Rodriguez, "Predictive control algorithm technique for multilevel asymmetric cascaded h-bridge inverters," Industrial Electronics, IEEE Transactions on, vol. 55, no. 12, pp. 4354 $-4361,2008$

[21] J. Song-Manguelle, S. Mariethoz, M. Veenstra, and A. Rufer, "A generalized design principle of a uniform step asymmetrical multilevel converter for high power conversion," in EPE 2001 European Conference on Power Electronics and Applications, Sept. 2001.

[22] P. Cortes, S. Kouro, B. La Rocca, R. Vargas, J. Rodriguez, J. Leon, S. Vazquez, and L. Franquelo, "Guidelines for weighting factors design in model predictive control of power converters and drives," in Industrial Technology, 2009. ICIT 2009. IEEE International Conference on, pp. 1 $-7,2009$.

[23] R. Vargas, U. Ammann, B. Hudoffsky, J. Rodriguez, and P. Wheeler, "Predictive torque control of an induction machine fed by a matrix converter with reactive input power control," Power Electronics, IEEE Transactions on, vol. 25, pp. 1426 -1438, june 2010.

[24] R. Vargas, U. Ammann, and J. Rodriguez, "Predictive approach to increase efficiency and reduce switching losses on matrix converters,' Power Electronics, IEEE Transactions on, vol. 24, no. 4, pp. 894 -902, 2009.

[25] C. Townsend, T. Summers, and R. Betz, "Comparison of modulation strategies for a cascaded h-bridge statcom - part 1: Theoretical background," in Industrial Electronics, 2010. IECON 2010. International Conference on, pp. 1-6, Nov. 2010.

[26] Y.-R. Mohamed and E. El-Saadany, "Robust high bandwidth discretetime predictive current control with predictive internal mode a; unified approach for voltage-source pwm converters," Power Electronics, IEEE Transactions on, vol. 23, no. 1, pp. $126-136,2008$.

[27] A. Al-Zamil and D. Torrey, "A passive series, active shunt filter for high power applications," Power Electronics, IEEE Transactions on, vol. 16, pp. $101-109$, Jan. 2001.

[28] S. Henriksen, "Digital predictive current control of induction machines," in Master's thesis, Dept. Elect. Comput. Eng., Univ. Newcastle, Callaghan, Australia, 2001.

[29] R. Betz, G. Mirzaeva, C. Townsend, and T. Summers, "Feed-forward compensation for multilevel cascaded h-bridge statcoms," in Power Electronics and Applications, 2009. EPE '09. 13th European Conference on, pp. 1-10, Sept. 2009. 